\title{
The comparative analysis of the multiplicative atom-bond connectivity and geometric-arithmetic indices in random polyphenyl and spiro chains
}

\author{
Zahid Raza ${ }^{1}$ \\ ${ }^{1}$ University of Sharjah
}

May 5, 2020

\begin{abstract}
The polyphenyl chains with $\$ \mathrm{n} \$$ hexagons are the special graphs of unbranched polycyclic aromatic hydrocarbons. The objective of this study is to find the expected values of the multiplicative version of the atomic-bond connectivity index and geometricarithmetic index of this class of special hydrocarbons. The average values of these two indices with respect to the set of all polyphenyl chains have been determined. Finally, the comparisons between the expected values of the aforementioned indices in the random polyphenyl and spiro chains, have been outlined.
\end{abstract}

\section{Hosted file}

Multiplicative ABC and GA.pdf available at https://authorea.com/users/301298/articles/431102the-comparative-analysis-of-the-multiplicative-atom-bond-connectivity-and-geometricarithmetic-indices-in-random-polyphenyl-and-spiro-chains 